\title{
Minimal Dose of Tranexamic Acid Is Effective in Reducing Blood Loss in Complex Spine Surgeries: A Randomized Double-Blind Placebo Controlled Study
}

\author{
Moslem Shakeri, Firooz Salehpour, Ghaffar Shokouhi, Kamkar Aeinfar, \\ Javad Aghazadeh, Farhad Mirzaei, Seyed Ahmad Naseri Alavi \\ Department of Neurosurgery, Tabriz University of Medical Sciences, Tabriz, Iran
}

Study Design: A randomized double-blind placebo controlled study.

Purpose: In the present study, we aimed to assess the efficacy of tranexamic acid (TXA) in reducing blood loss after laminectomy and posterolateral fusion of the spine.

Overview of Literature: Blood loss is the most significant complication involved with surgery, especially in spinal surgery. Multilevel laminectomy and laminectomy with instrumentation (pedicle screws and rods) are complex spine surgeries and are considered as medium-risk procedures for bleeding. Recent reports have demonstrated that the use of antifibrinolytic drugs during surgery may reduce the risk of postoperative bleeding and one of the most frequently used antifibrinolytics is TXA.

Methods: In this randomized clinical trial, 50 patients eligible for laminectomy (for $\geq 2$ level) with postero-lateral fusion with a pedicular screw (laminectomy and posterior spinal fusion) were randomly assigned to receive preoperative single doses of intravenous TXA (15 mg/kg) or $0.9 \%$ normal saline.

Results: Of the 50 patients, $30(60 \%)$ were female and $20(40 \%)$ were male. Between-group difference with respect to the total volume of blood loss during surgery was statistically significant.

Conclusions: The findings of this study suggest that TXA can reduce both intraoperative and immediate postoperative blood loss, decrease the need for packed cell transfusion, and reduce the duration of hospitalization after complex spinal surgeries. No adverse events related to the use of TXA were encountered in this study.

Keywords: Tranexamic acid; Antifibrinolytic agents; Laminectomy; Spine surgery; Blood loss

\section{Introduction}

Blood loss is still one of the most frequently occurring and significant complications of surgery, especially spinal surgery. Several studies have attempted reduction in blood loss and hemorrhage during and after spinal surgery [1-6]. Intraoperative and postoperative hemorrhage causes anemia, hematoma and necessitates blood transfusion, which may affect surgical outcomes [7]. In addition, blood transfusion increases the risk of immunological complications

Received Jul 8, 2017; Revised Aug 14, 2017; Accepted Aug 23, 2017

Corresponding author: Seyed Ahmad Naseri Alavi

Department of Neurosurgery, Tabriz University of Medical Sciences, Tabriz, Iran

Tel: +98-9116137353, Fax:+98-9116137353, E-mail: arsalan2010@gmail.com 
and mortality rate [8].

Perioperative antifibrinolytic therapy has been used for minimizing blood loss and reducing the need for blood transfusion [9]. Antifibrinolytics have been shown to reduce blood loss during cardiac and orthopedic surgeries $[10,11]$. Tranexamic acid (trans-4-aminomethyl-cyclohexane-1-carboxylic acid, TXA) is a high-potency synthetic antifibrinolytic drug that causes reversible competitive blockade of lysine-binding site of plasmin, plasminogen, and tissue plasminogen activator [12]. TXA has been shown to prevent platelet degradation by inhibiting plasmin and it also increases the formation of blood-clots [7].

The perioperative use of TXA was shown to prevent intraoperative blood loss in numerous studies $[1-6,13,14]$. The half-life of TXA is approximately 80 minutes and it is cleared via the kidney [15]. TXA is usually administered via oral, topical, and intravenous routes [15]; however, most studies have evaluated its use as an intravenous agent $[14,15]$. Studies of the perioperative use of TXA have employed a wide range of doses, which includes different bolus and infusion dosing; however, the suggested loading dose of TXA is $10-15 \mathrm{mg} / \mathrm{kg}[14,16]$. In a large multicenter study of 10,096 patients from 40 countries, the minimum effective dose of TXA was reported to be 15 $\mathrm{mg} / \mathrm{kg}$ [17].

The type and duration of procedure are the two most important determinants of blood loss that necessitate the need for transfusion during spinal surgery [16]. Multilevel laminectomy and laminectomy with instrumentation (pedicle screws and rods) are classified as complex spine surgeries and are considered as medium-risk procedures for bleeding and for the need for blood transfusion [16]. Raksakietisak et al. [16] used a double dose of $15 \mathrm{mg} / \mathrm{kg}$ of TXA during multi-level laminectomy and demonstrated a positive effect of TXA in reducing blood loss. The aim of this study was to assess the efficacy of TXA in reducing blood loss after laminectomy and posterolateral fusion of spine surgery.

\section{Materials and Methods}

\section{Study design}

Fifty patients were randomly divided into the following two groups: patients in the case group received preoperative single dose of intravenous TXA $15 \mathrm{mg} / \mathrm{kg}$ and the control group received only serum $0.9 \%$ normal saline
(NS) or placebo. Patients underwent surgery after the intravenous injection. The same surgeon performed laminectomy and posterior spinal fusion on all 50 patients using the same technique. No surgical and Gelfoam were used during the operation to rule out any confounding influence. After laminectomy and fusion, the surgical site, fascia, and muscles were packed with sterile gas. For achieving hemostasis at the end of surgery, arterial bleeding was controlled with coater and venous bleeding was arrested by packing with sterile gas. The number of sterile gases used during the surgery was recorded. Hemoglobin $\mathrm{Hb})$ was checked after admission and patients with $\mathrm{Hb}$ less than 7 received packed cell transfusion. All procedures were performed by the same team with the same method of surgery.

The two groups were compared with respect to age, sex, weight, body mass index (BMI), bleeding in the operation room, total volume of bleeding, bleeding volume in the first 12 hours after surgery, volume of bleeding between 12-24 hours after surgery, packed cells received, and hospitalization time.

This randomized double-blind clinical trial was approved by the Ethics Committee of Tabriz University of Medical Sciences (approval no., IR.TBZMED. REC.1395.1292). The study was performed in the department of neurosurgery at the Imam Reza Hospital of Tabriz University of Medical Sciences from March 2014 to March 2016. This clinical trial is registered in the Iranian Registration of Clinical Trial Center (IRCT) (IRCT no., IRCT2017020913947N6). Written informed consent was obtained from all patients or their accompanying family member/guardian prior to their enrollment.

\section{Inclusion criteria}

Fifty patients (age range, 18 to 60 years) participated in this study. These patients had either lumbar spinal stenosis or lumbar spondylolisthesis and were candidates for 2 or more than 2 levels of laminectomy and posterolateral fusion performed with instruments (pedicle screw and rods).

\section{Exclusion criteria}

Patients with a history of treatment with anticoagulant drugs, dipyridamole and oral contraceptives, those with abnormal international normalized ratio, prothrombin 
time and partial thromboplastin time, patients with cerebrovascular accident, myocardial infarction, coagulopathies, traumatic brain injury, cardiopulmonary resuscitation, renal failure, smoking, opioids, diabetes mellitus, hypertension, coronary artery disease, pregnant and breastfeeding women, and those who received packed cell transfusion during or after operation were excluded from the study.

\section{Randomization}

Fifty simple syringes prepared by the supervisor (one of the authors) were numbered from one to fifty. Twenty-five of these were filled with $15 \mathrm{mg} / \mathrm{kg}$ TXA and the remaining were filled with serum $0.9 \%$ NS. The syringes were delivered to the technician of the operating room for injection. The injections of TXA and NS were intravenously administered to patients in a blinded manner. After the surgery, the technician reported the name of the patients and the respective number of syringe used to the supervisor.

\section{Statistical analysis}

Data are presented as mean \pm standard deviation. Fisher's exact test or chi-square test (for categorical variables) and independent samples $t$-test (for numerical data) were used for assessing between-group differences. Statistical analyses were performed using SPSS ver. 16.0 software (SPSS Inc., Chicago, IL, USA). All $p$-values less than 0.05 were considered indicative of a statistically significant difference. Pearson's correlation coefficient ( $\mathrm{r}$ ) was calculated for assessing the correlation between whole blood loss and intra and postoperation and hospitalization, the correlation between BMI and blood loss, and the correlation between age and blood loss.

\section{Results}

Of the 50 patients, $20(40 \%)$ were male and $30(60 \%)$ were female (Table 1). The mean age of the patients was 49.82 \pm 7.87 years (range, $30-60$ years). The demographic, intraoperative, and postoperative characteristics in the two groups are presented in Table 1. Age, sex, and weight of patients was comparable in the two groups; however, a significant difference was noted between the two groups with respect to BMI, bleeding in the operation room, total volume of bleeding, bleeding volume in the first 12 hours and between 12-24 hours after surgery, packed cells received, and hospitalization time. A significant correlation was observed between the total volume of blood loss and duration of hospitalization in both case and control groups $(r=-0.44, p=0.02$ and $r=-0.72, p=0.001$, respectively) (Table 2). This correlation was stronger in the control group and demonstrated the positive effect of bleeding on the hospitalization period in the control group. There was no significant correlation between the total volume of bleeding and BMI in the case group $(r=-0.33, p=0.10)$. Also, there was no significant correlation noted between

Table 1. Comparison of the studied variables between the case and control groups

\begin{tabular}{|c|c|c|c|}
\hline Variable & Case group $(\mathrm{n}=25)$ & Control group (n=25) & $p$-value \\
\hline Sex & & & 0.16 \\
\hline Male & 12 & 8 & \\
\hline Female & 13 & 17 & \\
\hline Age (yr) & $50.52 \pm 6.51$ & $49.12 \pm 9.12$ & 0.59 \\
\hline Weight (kg) & $71.04 \pm 7.49$ & $71.44 \pm 1.82$ & 0.86 \\
\hline Body mass index & $26.68 \pm 2.52$ & $28.37 \pm 3.1$ & 0.47 \\
\hline Bleeding intra-operation (mL) & $261.60 \pm 106.91$ & $530.2 \pm 112.71$ & 0.0001 \\
\hline Bleeding in first $12 \mathrm{hr}(\mathrm{mL})$ & $80.60 \pm 7.27$ & $118.1 \pm 10.94$ & 0.014 \\
\hline Bleeding in second $12 \mathrm{hr}(\mathrm{mL})$ & $92.8 \pm 7.81$ & $134.2 \pm 14.35$ & 0.023 \\
\hline Whole amount of bleeding $(\mathrm{mL})$ & $632.2 \pm 193.133$ & $1037 \pm 242.61$ & 0.0001 \\
\hline Pack cell received (mL) & $440 \pm 583$ & $1320 \pm 748$ & 0.0001 \\
\hline Hospital stay (day) & $2.28 \pm 0.614$ & $3.36 \pm 1.11$ & 0.001 \\
\hline
\end{tabular}

Values are presented as number or mean \pm standard deviation. 
Table 2. Correlation between variables in case and control groups

\begin{tabular}{lcc} 
Variable & Pearson correlation (r) & $p$-value \\
\hline Amount of bleeding and hospitalization time (case group) & 0.44 & 0.02 \\
\hline Amount of bleeding and hospitalization time (control group) & 0.72 & 0.001 \\
\hline whole amount of bleeding and body mass index (case group) & -0.33 & 0.10 \\
Amount of bleeding and age in case group & -0.10 & 0.06 \\
\hline
\end{tabular}

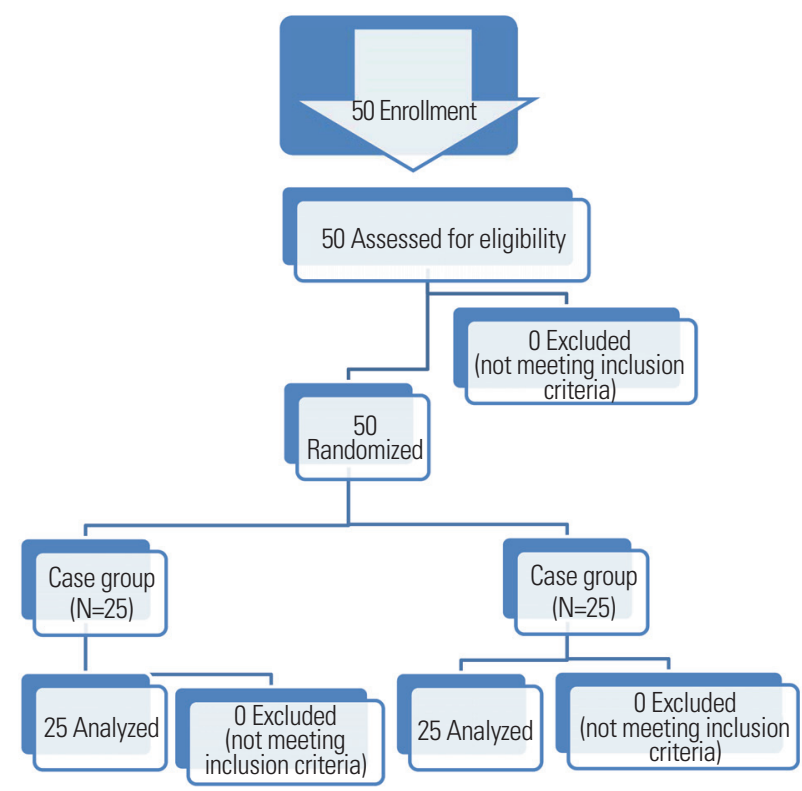

Fig 1. The flow chart of the study.

the total volume of blood loss and age in the case group $(r=-0.10, p=0.06)$. Fig. 1 displays the flowchart of the study.

\section{Discussion}

The aim of this study was to assess the efficacy of TXA in reducing blood loss after laminectomy with posterolateral fusion of the spine. There was no statistically significant difference noted between the study groups with respect to age, weight, and BMI. We found that use of TXA in the case group significantly reduced the hospitalization time and total volume of blood loss as compared with that in the control group.

Numerous studies have recommended different methods for reducing blood loss during spinal surgery and especially in the context of complex spine surgeries that are associated with a higher risk of bleeding. These include controlled hypotension, autologous blood transfusion, and the use of erythropoietin and antifibrinolytic drugs $[13,16,18]$. Intraoperative blood loss depends on many factors such as physical status, bodyweight, surgical technique, the number of segments involved, the skill set of the surgeon, the duration of operation, and individual patient characteristics.

TXA is an significant antifibrinolytic drug that has been used in different fields of surgery such as orthopedics and spine surgery; however, the effective dose is still unclear $[16,18]$. In order to minimize the risk of complications, we opted for a preoperative single loading dose of $15 \mathrm{mg} /$ $\mathrm{kg}$ without infusion. The minimum safe dose of TXA was reported to be $15 \mathrm{mg} / \mathrm{kg}$ [17]; however, another recent study demonstrated the safety and efficacy of twice the minimum safe dose [16]. In this study, a single dose of TXA was used and our results demonstrate a positive effect of TXA. In addition, no side effects were recorded. Raksakietisak et al. [16] used 2 doses of $15 \mathrm{mg} / \mathrm{kg}$ TXA (before and 3 hours after surgery, respectively). The total volume of blood loss in the case group $(1,050 \mathrm{~mL})$ was higher than that in the present study $(632 \mathrm{~mL})$. Shi et al. [19] used a loading dose of $30 \mathrm{mg} / \mathrm{kg}$ TXA followed by the infusion of $2 \mathrm{mg} / \mathrm{kg} / \mathrm{hr}$; however, the total volume of blood loss in the case group was lower as compared with that in the present study ( $477 \mathrm{~mL}$ versus $632 \mathrm{~mL}$ ). Ngo et al. [9] used a loading dose of $50 \mathrm{mg} / \mathrm{kg}$ TXA followed by an infusion of $5-10 \mathrm{mg} / \mathrm{kg} / \mathrm{hr}$ in their study and reported a successful experience; however, blood loss in the operation room was higher than that observed in our study (437 $\mathrm{mL}$ versus $261 \mathrm{~mL}$ ). None of the above-mentioned studies reported any adverse events associated with the different doses of TXA $[9,16,19]$.

Our results are comparable with those of other studies. Therefore, it can be concluded that higher doses of TXA have no significant effect in reducing blood loss associated with complex spine surgery $[9,16,19]$. In the study by Raksakietisak et al. [16], 11 patients received packed cells in the intra- and postoperative period, which is higher than 
that observed in our study (nine patients received 1 unit of packed cells and one patient received 2 units of packed cells). In the study by Ngo et al. [9], the volume of packed cells received in the case group was $298 \mathrm{~mL}$, which was lower than that in our study $(440 \mathrm{~mL})$. Therefore, higher doses of TXA seem to decrease the need for packed cells and can thus be more effective than a double single dose of TXA.

We also investigated the correlation between the total volume of blood loss and duration of hospitalization in both groups and also assessed the effect of bleeding on hospitalization. No significant correlation of the total volume of blood loss with age or BMI was observed in the case group. However, a significant correlation was observed between the total volume of bleeding and hospitalization in the case group.

\section{Conclusions}

The findings of this study suggest that TXA can reduce bleeding amount in the first and second 12 hours after surgery, the total volume of intraoperative blood loss, the total volume of blood loss, the duration of hospitalization, and requirement for packed cells without causing any adverse events. On the contrary, we found that the effect of minimum dose of TXA in reducing blood loss was comparable with that of higher doses.

\section{Conflict of Interest}

No potential conflict of interest relevant to this article was reported.

\section{References}

1. Neilipovitz DT, Murto K, Hall L, Barrowman NJ, Splinter WM. A randomized trial of tranexamic acid to reduce blood transfusion for scoliosis surgery. Anesth Analg 2001;93:82-7.

2. Sethna NF, Zurakowski D, Brustowicz RM, Bacsik J, Sullivan LJ, Shapiro F. Tranexamic acid reduces intraoperative blood loss in pediatric patients undergoing scoliosis surgery. Anesthesiology 2005;102:727-32.

3. Wong J, El Beheiry H, Rampersaud YR, et al. Tranexamic acid reduces perioperative blood loss in adult patients having spinal fusion surgery. Anesth Analg 2008;107:1479-86.
4. Shapiro F, Zurakowski D, Sethna NF. Tranexamic acid diminishes intraoperative blood loss and transfusion in spinal fusions for duchenne muscular dystrophy scoliosis. Spine (Phila Pa 1976) 2007;32:227883.

5. Yagi M, Hasegawa J, Nagoshi N, et al. Does the intraoperative tranexamic acid decrease operative blood loss during posterior spinal fusion for treatment of adolescent idiopathic scoliosis? Spine (Phila Pa 1976) 2012;37:E1336-42.

6. Elwatidy S, Jamjoom Z, Elgamal E, Zakaria A, Turkistani A, El-Dawlatly A. Efficacy and safety of prophylactic large dose of tranexamic acid in spine surgery: a prospective, randomized, double-blind, placebo-controlled study. Spine (Phila Pa 1976) 2008;33:2577-80.

7. Winter SF, Santaguida C, Wong J, Fehlings MG. Systemic and topical use of tranexamic acid in spinal surgery: a systematic review. Global Spine J 2016;6:284-95.

8. Marik PE, Corwin HL. Efficacy of red blood cell transfusion in the critically ill: a systematic review of the literature. Crit Care Med 2008;36:2667-74.

9. Ngo KT, Khemani RG, Choi PD, Ross PA. Tranexamic acid reduces PRBC transfusion after posterior spine surgery for idiopathic scoliosis from the operating room to post-operative day four. J Anesthe Clin Res 2013;4:7. https://doi.org/10.4172/21556148.1000338 .

10. Brown JR, Birkmeyer NJ, O'Connor GT. Meta-analysis comparing the effectiveness and adverse outcomes of antifibrinolytic agents in cardiac surgery. Circulation 2007;115:2801-13.

11. Alshryda S, Sarda P, Sukeik M, Nargol A, Blenkinsopp J, Mason JM. Tranexamic acid in total knee replacement: a systematic review and meta-analysis. J Bone Joint Surg Br 2011;93:1577-85.

12. Hardy JF, Desroches J. Natural and synthetic antifibrinolytics in cardiac surgery. Can J Anaesth 1992;39:353-65.

13. Yang B, Li H, Wang D, He X, Zhang C, Yang P. Systematic review and meta-analysis of perioperative intravenous tranexamic acid use in spinal surgery. PLoS One 2013;8:e55436.

14. Neilipovitz DT. Tranexamic acid for major spinal surgery. Eur Spine J 2004;13 Suppl 1:S62-5.

15. Horrow JC, van Riper DF, Strong MD, Grunewald 
KE, Parmet JL. The dose-response relationship of tranexamic acid. Anesthesiology 1995;82:383-92.

16. Raksakietisak M, Sathitkarnmanee B, Srisaen P, et al. Two doses of tranexamic acid reduce blood transfusion in complex spine surgery: a prospective randomized study. Spine (Phila Pa 1976) 2015;40:E125763.

17. CRASH-2 trial collaborators, Shakur H, Roberts I, et al. Effects of tranexamic acid on death, vascular occlusive events, and blood transfusion in trauma patients with significant haemorrhage (CRASH-2): a randomised, placebo-controlled trial. Lancet 2010;376:2332.
18. Li ZJ, Fu X, Xing D, Zhang HF, Zang JC, Ma XL. Is tranexamic acid effective and safe in spinal surgery?: a meta-analysis of randomized controlled trials. Eur Spine J 2013;22:1950-7.

19. Shi H, Ou Y, Jiang D, Quan Z, Zhao Z, Zhu Y. Tranexamic acid reduces perioperative blood loss of posterior lumbar surgery for stenosis or spondylolisthesis: A randomized trial. Medicine (Baltimore) 2017;96:e5718. 